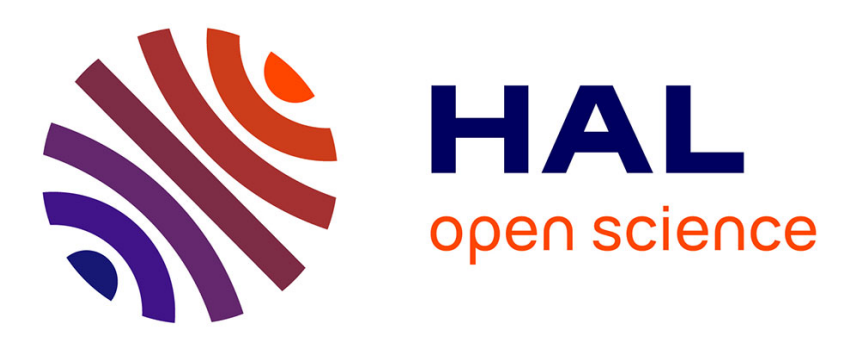

\title{
Réalisation d'un spectromètre infrarouge pour l'analyse comparative de l'émissivité directionnelle des surfaces
}

\author{
$\mathrm{Ph}$. Pigeat, D. Paulmier
}

\section{To cite this version:}

Ph. Pigeat, D. Paulmier. Réalisation d'un spectromètre infrarouge pour l'analyse comparative de l'émissivité directionnelle des surfaces. Revue de Physique Appliquée, 1982, 17 (6), pp.393-398. 10.1051/rphysap:01982001706039300 . jpa-00245012

\section{HAL Id: jpa-00245012 https://hal.science/jpa-00245012}

Submitted on 1 Jan 1982

HAL is a multi-disciplinary open access archive for the deposit and dissemination of scientific research documents, whether they are published or not. The documents may come from teaching and research institutions in France or abroad, or from public or private research centers.
L'archive ouverte pluridisciplinaire HAL, est destinée au dépôt et à la diffusion de documents scientifiques de niveau recherche, publiés ou non, émanant des établissements d'enseignement et de recherche français ou étrangers, des laboratoires publics ou privés. 


\title{
Réalisation d'un spectromètre infrarouge pour l'analyse comparative de l'émissivité directionnelle des surfaces
}

\author{
Ph. Pigeat et D. Paulmier \\ Laboratoire d'Etudes et Recherches en Mécanique et Energétique des Surfaces, E.R.A. 892, \\ 2, rue de la Citadelle, 54000 Nancy, France \\ (Reçu le ler octobre 1981, accepté le 26 février 1982)
}

\begin{abstract}
Résumé. - Nous décrivons un spectromètre infrarouge parfaitement adapté à la mesure de faibles variations dans l'émissivité monochromatique directionnelle des surfaces métalliques. Le spectre étudié s'étend de $0,5 \mu \mathrm{m}$ à $10 \mu \mathrm{m}$ pour des températures d'échantillon situées entre $400 \mathrm{~K}$ et $1000 \mathrm{~K}$. L'excellente résolution relative même pour des facteurs radiatifs faibles $(4 \%$ à $\varepsilon=0,2$ pour $2 \mu \mathrm{m}<\lambda<4 \mu \mathrm{m})$ est obtenue en comparant l'énergie émise par les faces d'un ruban métallique. Dans cette configuration, la différence en température $\left(<10^{-3} \mathrm{~K}\right)$ entre les deux échantillons à comparer devient négligeable. L'ensemble complet, source-détecteur, est monté sous ultra vide. Le système optique assure la comparaison des énergies émises sous des angles solides et des angles d'émission rigoureusement identiques. De plus cet appareil est suffisamment pratique pour permettre la mesure systématique de faibles variations d'émissivité conséquence, par exemple, d'une faible modification d'un paramètre de surface comme la rugosité ou l'épaisseur d'un dépôt.
\end{abstract}

\begin{abstract}
An infrared spectrometer perfectly adapted to the measurement of small variations in spectral and directional emissivity of metallic surfaces is presented. The accessible wavelengths range from $0.5 \mu \mathrm{m}$ to $10 \mu \mathrm{m}$ at temperatures between $400 \mathrm{~K}$ and $1000 \mathrm{~K}$. The excellent relative resolution is obtained comparing radiative power of each sides of a metallic ribbon ( $4 \%$ at $\varepsilon=0.2$ for $2 \mu \mathrm{m}<\lambda<4 \mu \mathrm{m})$. In this configuration the difference between the temperatures $\left(10^{-3} \mathrm{~K}\right)$ of the two samples becomes negligible. The complete optic assembly including the source and the detector is maintained under high vacuum. The optical system assure the comparison of emerging power under rigourously identical solid angles and angles of emission. Furthermore, this device is fairly convenient to allow a systematic measurement from a small variations in emissivity like those resulting from a change of surface parameters such as the roughness or the thickness of a superficial film.
\end{abstract}

1. Introduction - La dispersion des résultats publiés sur les facteurs optiques [1], ainsi que le manque d'informations précises quant aux caractéristiques des échantillons attachés à ces résultats rendent ces derniers difficilement utilisables dans certains domaines de recherche. Ce manque est particulièrement important lorsqu'il s'agit des facteurs d'émissivité $\varepsilon$. En conversion photothermique de l'énergie solaire, en pyrométrie, et chaque fois que le rayonnement est envisagé comme moyen d'information, l'utilisateur ne possède pas de résultats, de relations phénoménologiques et de théories suffisamment précises pour contrôler, prévoir, et donc maîtriser les propriétés radiatives d'une surface. Les techniques d'études du facteur de réflexion sont aujourd'hui très développées et il serait tentant de les utiliser pour calculer l'émissivité d'un corps; toutefois, les résultats théoriques ou expérimentaux obtenus de cette manière (méthode indirecte) nécessitent plusieurs intégrations successives et l'incertitude sur le résultat final est importante [2], [3], [4]. Seule une mesure directe des caractéristiques radiatives d'une surface peut donner une précision suffisante. Certaines expériences très soignées faites dans ces conditions ont donné des résultats exploitables [5], mais elles sont rares et beaucoup reste encore à faire. Une étude convenable de l'émissivité monochromatique directionnelle d'une surface par une méthode directe, demande, outre la mise en ouvre de techniques permettant de caractériser la surface analysée [6] à [11] (microprofilomètre, microscope à balayage, spectrométrie Auger, etc.), la mise au point d'un spectromètre qui satisfasse au moins les quatre conditions suivantes :

- Grande sensibilité et minimisation des absorptions parasites (pour le cas où les énergies mesurées sont très faibles).

- Egalité des conditions expérimentales lors des 
mesures faites sur l'échantillon et sur la source de référence.

- Stabilité des caractéristiques physico-chimiques des surfaces pendant le temps nécessaire aux mesures.

- Egalité des températures des sources comparées.

Aucun système expérimental proposé antérieurement ne satisfait pleinement ces exigences. La dernière de ces quatre conditions n'est notamment jamais suffisamment bien réalisée alors qu'elle entraîne à elle seule des erreurs particulièrement importantes sur le facteur d'émission (Fig. 1) [3], [4].

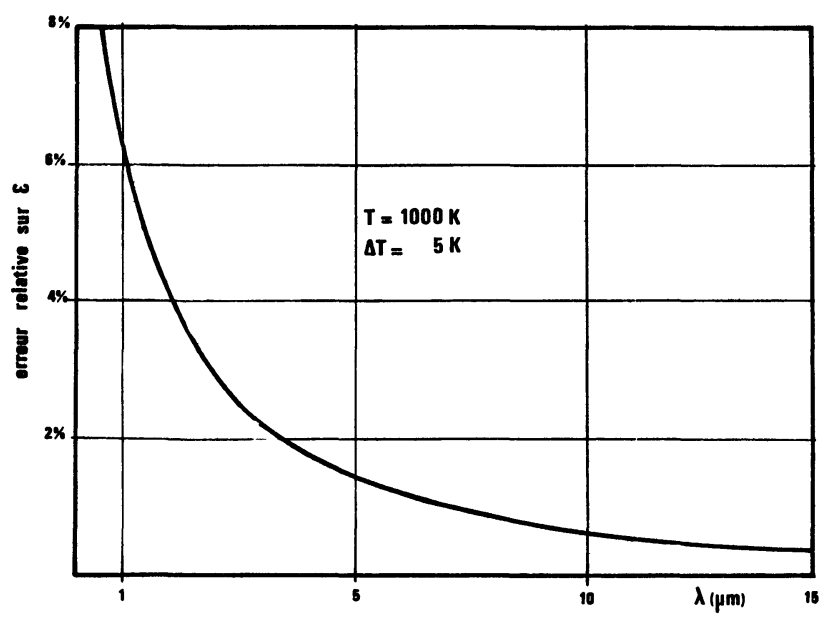

Fig. 1. - Erreur relative sur l'émissivité d'un corps à $1000 \mathrm{~K}$ lorsque la différence de température entre la surface étudiée et la surface référence (ici un corps noir) est de $5 \mathrm{~K}$.

[Relative error in the emissivity of a sample admitting a difference of $5 \mathrm{~K}$ between the surface temperatures of sample and reference source.]

Le spectromètre mis au point au Laboratoire E.R.M.E.S. et décrit dans cet article satisfait ces conditions.

2. Optique. - La méthode expérimentale classique pour l'étude directe de l'émissivité consiste à comparer la luminance d'un corps noir à la luminance d'une surface échantillon $\mathrm{A}$, puis dans un second temps à celle d'une surface échantillon B. Nous avons abandonné cette technique. Certes, la comparaison des

Fig. 2. - Schéma du support source. 1) Socle de l'enceinte à vide, contenant l'ensemble du spectrophotomètre. 2) Chapeau de l'enceinte. 3) Arbre permettant la rotation du ruban (étanchéité assurée par presse-étoupe sur (2)). 4) Double paroi absorbant le rayonnement parasite issu du ruban. 5) Ouverture symétrique dans la double paroi pour l'observation du ruban par le double circuit. 6) Ruban échantillon. 7) Prise de potentiel microsoudé au ruban. 8) Enroulement de résistance chauffante pour thermostater les amenées de courants (11) (9). 9) Amenée de courant mobile (absorption de la dilatation du ruban). 10) Cône fixe. 11) Amenée de courant fixe (réglable en hauteur [comme (9)] sur les tiges (15) par l'intermédiaire des bagues (14), pour permettre le montage de ruban de

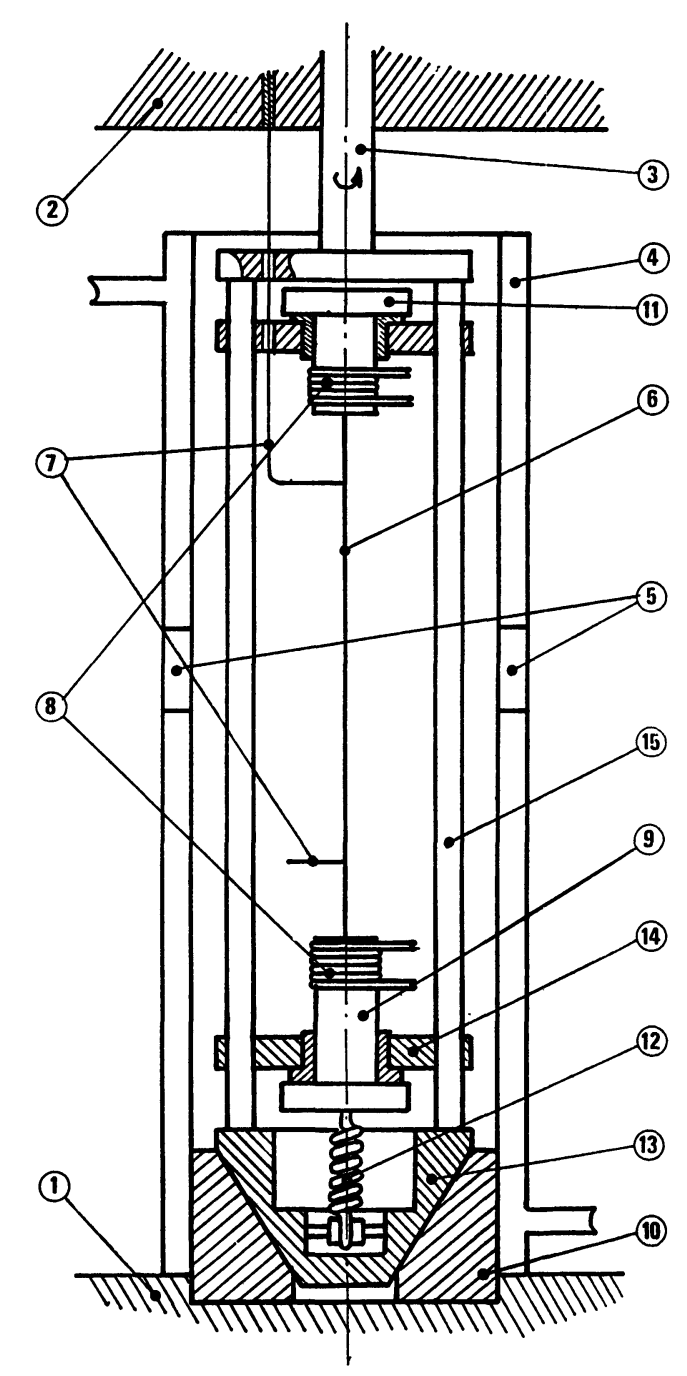

diverses longueurs). 12) Ressort de rappel du mors (9) absorbe la dilatation du ruban. 13) Cône mâle mobile permettant la rotation du support pour l'étude des indicatrices d'émission $\theta=f(\theta)$. 14) Bagues-support des amenées de courant (9) et (11). 15) Tige d'inox servant de glissière aux bagues (14) et placée de telle façon qu'elle ne diaphragme pas les faisceaux étudiés issus du ruban.

[Specimen monting. 1) Base of the vacuum chamber containing the complete spectrometer. 2) Mobile vacuum bell jar. 3) Axle allowing for a rotation of the ribbon specimen (O-ring vacuum-tight feed-through). 4) Double shielding absorbing stray radiation from the specimen. 5) Symmetric apertures into the double shielding for observation of the two beams emerging from both sides of the ribbonspecimen. 6) Ribbon-specimen. 7) Potential leads, spot welded on to the specimen. 8) Heating coils to reduce thermal leaks along the current leads (9) and (11). 9) Mobile current lead. 10) Fixed cone. 11) Fixed current lead (for specimens position adjustement of different length by means by rings sliding (14) along the rods (15)). 12) Spring on the mobile grips (9) absorbing the specimen's thermal expansion. 13) Mobile cone allowing for a rotation of the sample-holder in case of directional studies $\varepsilon=f(\theta)$. 14) Annular fixtures of the current leads (9) and (11). 15) Stainless steel slide-bar for (14) positioned such as to ovoid any interference with the beams of radiation, emerging from the specimen.] 
émissivités absolues $\varepsilon_{\mathrm{A}}$ et $\varepsilon_{\mathrm{B}}$ permet d'obtenir le sens de la variation du comportement radiatif engendrée par des différences microgéométriques, physiques ou chimiques entre ces surfaces, mais présente l'inconvénient d'être peu précise lorsque les luminances de $\mathbf{A}$ et de $\mathbf{B}$ sont peu différentes ou faibles devant celle du corps noir.

2.1 SoURCE (Fig. 2). - Dans notre spectromètre, l'intermédiaire corps noir est supprimé. Un ruban métallique de faible épaisseur et chauffé par effet Joule, matérialise par ses deux faces les échantillons A et $B$ à comparer (par exemple : deux faces du métal nu, mais de rugosités différentes; ou, la surface nue d'un côté et, un dépôt en oxyde, ou le substrat dopé sur l'autre). L'originalité de ce montage est de comparer des énergies du même ordre de grandeur, émises par des surfaces qui, vu l'épaisseur du ruban (quelque $10^{-4} \mathrm{~m}$ ) peuvent être considérées à la même température. La valeur de cette température est obtenue à partir de la mesure de résistivité du ruban entre deux prises de potentiel soudées. Le ruban est tendu verticalement entre deux mors faisant office d'amenée de courant. Ces mors réglables en hauteur pour le montage de ruban de longueur différente (jusqu'à $150 \mathrm{~mm}$ ) sont eux-mêmes chauffés par des enroulements de résistances chauffantes et minimisent ainsi les pertes thermiques par conduction au travers des extrémités. On vérifie que la zone étudiée optiquement peut être considérée comme isotherme. Le mors inférieur pourvu d'un ressort, rattrape la dilatation thermique et oblige le ruban à rester dans le plan source du système optique. La partie conique à la base du support de ruban permet, outre un démontage facile et un positionnement précis, la rotation du ruban autour de son grand axe.

2.2 Circuit OPTIQUE. - Les rayonnements A et $B$ issus des deux faces suivent un circuit doubles faisceaux (Fig. 3). Deux séries de miroirs plans $A_{1}$; $A_{2} ; A_{3}$; d'une part et $B_{1} ; B_{2} ; B_{3}$; d'autre part, orientent les faisceaux $A$ et $B$ vers un miroir concave $\mathrm{AB}_{1}$ commun aux deux circuits. $\mathrm{B}_{3}$ est un miroir tournant, constitué d'un disque ouvert sur un quadrant pour laisser passer le faisceau $A$ et métallisé sur le quadrant opposé pour réfléchir le faisceau B. La rotation de $B_{3}$ permet d'obtenir successivement, sur la fente d'entrée $F_{1}$ d'un monochromateur à prisme $\mathrm{NaCl}$, l'image des surfaces $A$ et $B$ séparées par une obturation complète. Le trajet $F_{1}, A_{1}$ est commun aux deux circuits, le reste des chemins optiques jusqu'au ruban étant de longueur égale, les sources A et $B$ sont alors étudiées obligatoirement sous le même angle solide, le même grandissement et avec la même distorsion des images. Le fait que chaque circuit comporte le même nombre de miroirs, entraîne l'égalité des coefficients de transmission et, l'alignement des miroirs $A_{1}$ et $B_{1}$ avec l'axe de rotation du ruban impliquera systématiquement l'égalité des angles

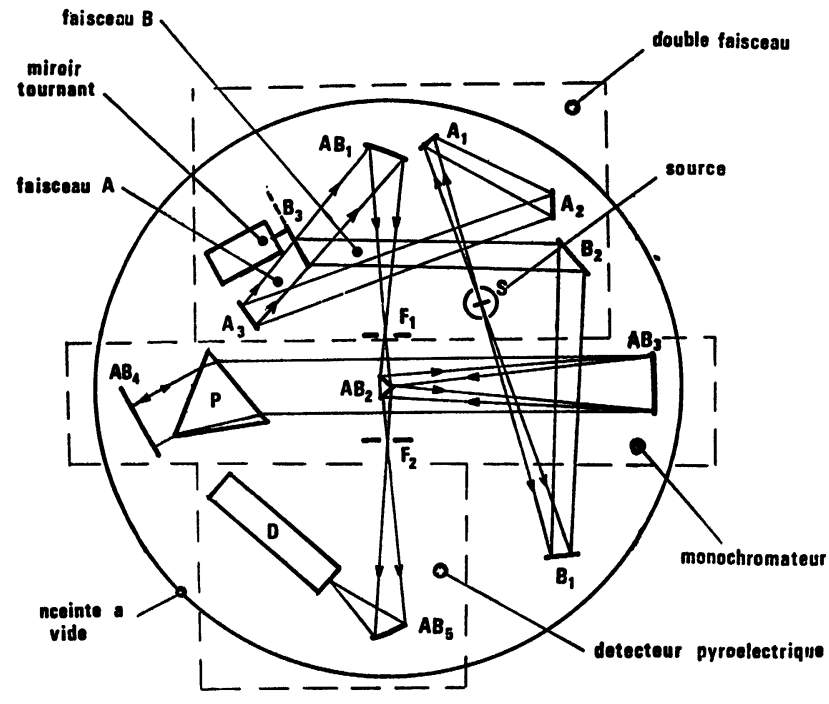

Fig. 3. - Schéma (vue de dessus) de l'enceinte et du spectrophotomètre qu'elle contient.

[Schematic view of the vacuum chamber and the spectrometer.]

d'émission $\theta_{\mathrm{A}}$ et $\theta_{\mathrm{B}}$ lors de l'étude comparative des indicatrices $\varepsilon(\theta)$.

Un monochromateur à prisme $\mathrm{NaCl}$ du type «LITTROW » associé à un détecteur pyroélectrique PLESSEY - PLT-222 - analyse les énergies émises par les faces du ruban sur une plage spectrale allant de 0,5 à $10 \mu \mathrm{m}$.

Cet ensemble, source, double faisceau, monochromateur, détecteur a été optimisé afin d'occuper un volume minimum et est réunie sur un plateau circulaire de diamètre $500 \mathrm{~mm}$. Une cloche vient recouvrir ce spectromètre et permet de travailler sous vide $\left(10^{-7}\right.$ torr) ou sous atmosphère contrôlée.

Cette configuration permet de protéger les échantillons contre toute modification due au milieu gazeux et présente l'avantage d'éviter les absorptions parasites le long des trajets optiques (absorption par $\mathrm{H}_{2} \mathrm{O}$ et $\mathrm{CO}, \mathrm{CO}_{2}$ de l'air, ou par des fenêtres intercalées entre la source et le spectromètre).

3. Analyse du signal (Figs. 4, 5). - Le miroir tournant $B_{3}$ sélectionne les faisceaux $A$ et $B$ à une fréquence de $0,2 \mathrm{~Hz}$ (Fig. 5a, haut). Les signaux $S_{a}$ et $S_{b}$ de deux photodiodes placées sur $B_{3}$ permettent de différencier le faisceau A du faisceau B (Fig. $5 a$, bas). Un hacheur $(19 \mathrm{~Hz})$ est placé devant la fenêtre d'entrée du monochromateur, le signal lumineux ainsi modulé (Fig. 5b), traduit électriquement par le capteur pyroélectrique (Fig. 5c), est mesuré par un amplificateur à détection synchrone PAR 128 A (Fig. 5d). Cette détection est synchronisée à la fréquence $19 \mathrm{~Hz}$ grâce à une diode placée sur le hacheur. Des portes électroniques commandées par $S_{a}$ et $S_{b}$ orientent le signal ainsi filtré vers deux circuits intégrateur échantillonneur bloqueur $\left(\mathrm{C}_{\mathrm{A}}, \mathrm{C}_{\mathrm{B}}\right.$ Fig. 4). Les valeurs $E_{\mathrm{A}}$ et $E_{\mathrm{B}}$ des signaux issus de ces deux circuits sont proportionnels aux 


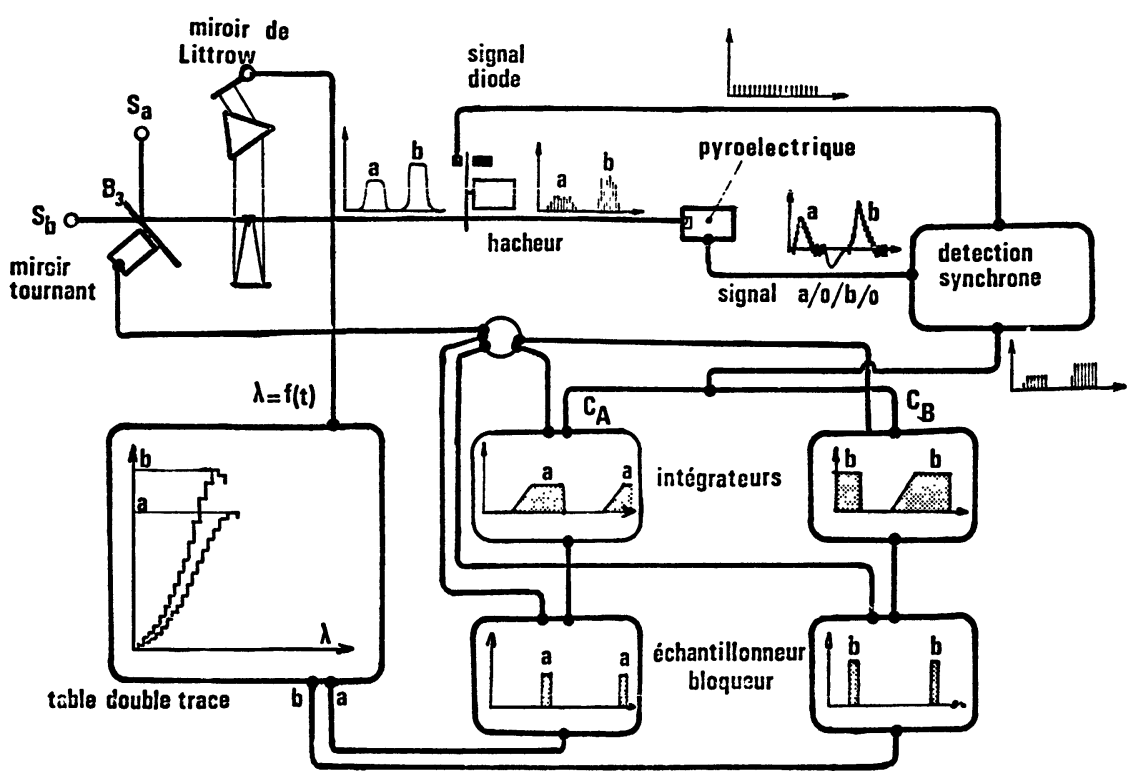

Fig. 4. - Schéma de principe du traitement du signal.

[Schematic diagram of the optics and electronics used for measurement.]

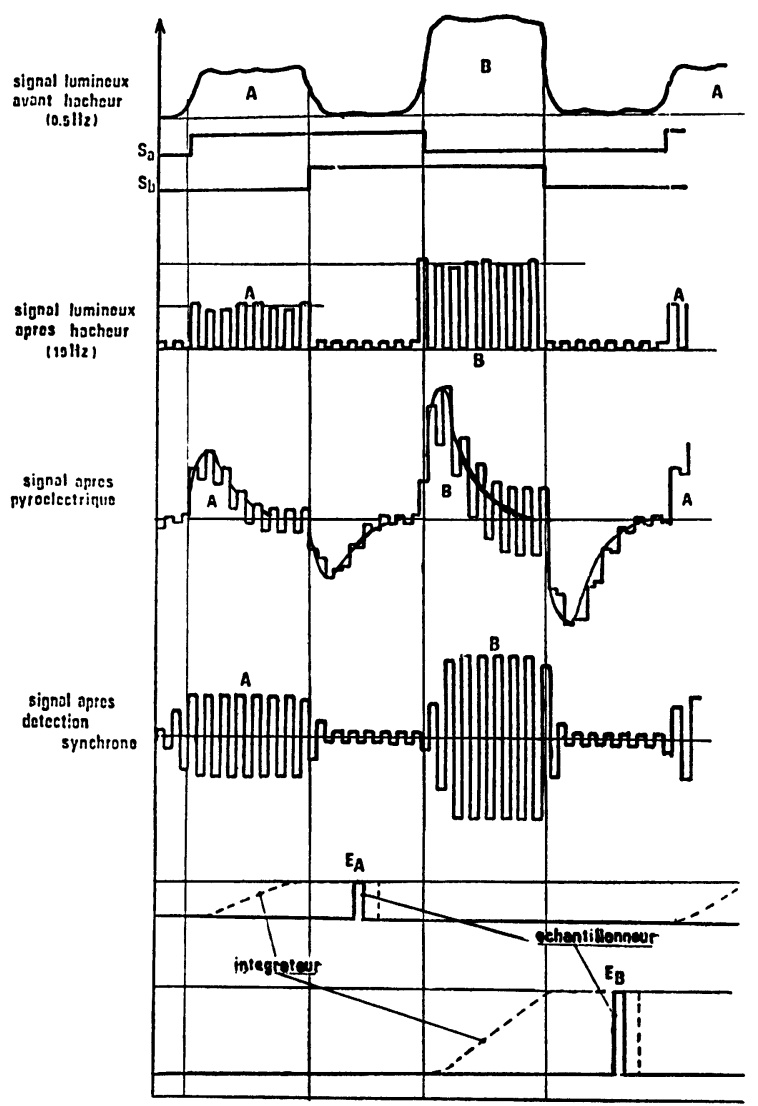

Fig. 5. - Forme des signaux à différents stades de la mesure.

[Shape of the signal in the course of experiment.]

énergies lumineuses émises des surfaces $A$ et $B$ pendant le temps d'ouverture $\Delta t=0,5 \mathrm{~s}$ imposé par le miroir $\mathrm{B}_{3}$. Ces valeurs $E_{\mathrm{A}}$ et $E_{\mathrm{B}}$ peuvent être enregis- trées sur une table traçante avec en abscisse le déplacement automatique et continu du miroir de «LITTROW ", soit en valeur absolue pour obtenir des spectres caractéristiques de $\mathrm{A}$ et $\mathrm{B}$, soit (grâce à un diviseur) sous forme de rapport

$$
k_{\lambda}=\frac{E_{\mathrm{A}}}{E_{\mathrm{B}}} .
$$

Ce rapport $k_{\lambda}$ traduit en fait le rapport des émissivités $\varepsilon_{\mathrm{A} \lambda \theta} / \varepsilon_{\mathrm{B} \lambda \theta}$, émissivités monochromatiques directionnelles des surfaces $\mathrm{A}$ et $\mathrm{B}$. $T_{\mathrm{A}}$ et $T_{\mathrm{B}}$ étant les températures des surfaces $\mathrm{A}$ et $\mathrm{B}$ et $L_{0 \lambda T}$ la luminance du corps noir à la longueur d'onde $\lambda$ et à la température $T$, le rapport $k_{\lambda}$ s'écrit :

$$
k_{\lambda}=\frac{E_{\mathrm{A}}}{E_{\mathrm{B} \lambda}}=\frac{\mathrm{d} S_{\mathrm{A}} \mathrm{d} \Omega_{\mathrm{A}} \tau_{\mathrm{A}}^{\prime}}{\mathrm{d} S_{\mathrm{B}} \mathrm{d} \Omega_{\mathrm{B}} \tau_{\mathrm{B}}} \frac{\int_{\Delta \lambda} \varepsilon_{\mathrm{A} \lambda \theta} L_{0 \lambda T_{\mathrm{A}}} \mathrm{d} \lambda}{\int_{\Delta \lambda} \varepsilon_{\mathrm{B} \lambda \theta} L_{0 \lambda T_{\mathrm{B}}} \mathrm{d} \lambda} .
$$

Le montage impose l'égalité des angles d'émission $\theta_{\mathbf{A}}=\theta_{\mathbf{B}}=\theta$. Si le circuit double faisceau est correctement réglé, leur coefficient de transmission $\tau_{\mathrm{A}}$ et $\tau_{\mathrm{B}}$, leurs angles solides $\mathrm{d} \Omega_{\mathrm{A}}$ et $\mathrm{d} \Omega_{\mathrm{B}}$ et les surfaces de ruban effectivement étudiées par l'appareil $\mathrm{d} S_{\mathrm{A}}$ et $\mathrm{d} S_{\mathrm{B}}$ sont égaux. Le rapport $k_{\lambda}$ se réduit donc à :

$$
k_{\lambda}=\frac{E_{\mathrm{A}}}{E_{\mathrm{B} \lambda}}=\frac{\int_{\Delta \lambda} \varepsilon_{\mathrm{A} \lambda \theta} L_{0 \lambda T_{\mathrm{A}}} \mathrm{d} \lambda}{\int_{\Delta \lambda} \varepsilon_{\mathrm{B} \lambda \theta} L_{0 \lambda T_{\mathrm{B}}} \mathrm{d} \lambda} .
$$

En choisissant une vitesse de défilement du miroir de « LITTROW » suffisamment lente pour que la varia- 
tion de longueur d'onde entre deux mesures consécutives $E_{\mathrm{A}}, E_{\mathrm{B}}$ soit négligeable, le rapport devient finalement

$$
k_{\lambda}=\left(\frac{E_{\mathrm{A}}}{E_{\mathbf{B}}}\right)_{\lambda}=\left(\frac{\varepsilon_{\mathrm{A}}}{\varepsilon_{\mathbf{B}}}\right)_{\theta, \lambda}
$$

la faible épaisseur du ruban permettant quant à elle de négliger la différence $T_{A}-T_{B}(\S 4.2)$.

4. Erreurs systématiques. - 4.1 MeSURE DE LA TEMPÉRATURE ET HOMOGÉNÉITÉ SUR LA ZONE ÉTUDIÉE. L'incertitude sur la mesure de la résistivité relative du ruban est d'environ $0,3 \%$. La comparaison de cette mesure avec une courbe d'étalonnage permet d'obtenir la température $T$ de la zone étudiée, avec une précision de $1,3 \%$ à $1000 \mathrm{~K}$.

En vue de vérifier l'homogénéité de la température sur la zone étudiée par le spectrophotomètre $(25 \mathrm{~mm})$, nous avons soudé quatre prises de potentiel à différentes distances d'un des mors. Les amenées de courant sont thermostatées à une température $T_{\mathrm{M}}$. En admettant que le gradient de température dû à la conduction à travers les prises de potentiel soit symétrique, il est aisé de corriger l'erreur de température qu'elles entraînent. Il a ainsi été vérifié théoriquement [12] et expérimentalement [3] sur un ruban d'acier inoxydable ASI 304, que pour différentes températures $T_{\mathrm{M}}$ et pour un échantillon de $150 \mathrm{~mm}$ de longueur par exemple, il existe une zone toujours largement supérieure à la zone radiative étudiée et dont le gradient $\Delta T$ inférieur à $1 \%$ pour $T<1000 \mathrm{~K}$, est suffisamment faible pour être négligé.

4.2 ECART DE TEMPÉRATURE ENTRE LES DEUX FACES DU RUBAN. - Dans la zone centrale du ruban (zone où le gradient de température longitudinale est négligeable), on considère un élément de volume $\Delta V$ pris transversalement, dont les pertes thermiques sont des pertes strictement radiatives par les faces A et $\mathbf{B}$.

(Le ruban est chauffé sous vide.) Le bilan énergétique de cet élément de volume, lorsque les émissivités totales hémisphériques $\varepsilon_{\mathrm{A}}$ et $\varepsilon_{\mathrm{B}}$ des faces $\mathrm{A}$ et $\mathrm{B}$ sont différentes, conduit à résoudre l'équation suivante : avec

$$
w l-\sigma \varepsilon_{\mathrm{A}} T_{\mathrm{A}}^{4}=\varepsilon_{\mathrm{B}} \sigma\left[\frac{\sigma \varepsilon_{\mathrm{A}} T_{\mathrm{A}}^{4}}{K} l-\frac{w l^{2}}{2 K}+T_{\mathrm{A}}\right]^{4}
$$

$$
\Delta T=T_{\mathrm{B}}-T_{\mathrm{A}}=\frac{\sigma \varepsilon_{\mathrm{A}} T_{\mathrm{A}}^{4}}{K} l-\frac{w l^{2}}{2 K}
$$

$-w, K$ l'énergie thermique créée au sein du matériau par unité de volume par effet Joule, et la conductivite thermique du matériau toutes deux supposées uniforme dans l'élément de volume $\Delta V$.

- $T_{\mathrm{A}}$ et $T_{\mathrm{B}}$ température des surfaces $\mathrm{A}$ et $\mathrm{B}$.

- $l$ épaisseur du ruban.

Pour un ruban, $K=293 \mathrm{~W} / \mathrm{mK}$, d'épaisseur $10^{-4} \mathrm{~m}$ et en imaginant des émissivités très différentes sur les deux faces $\varepsilon_{\mathrm{A}}=0,5$ et $\varepsilon_{\mathrm{B}}=0,25$ soit $\Delta \varepsilon / \varepsilon=50 \%$, les différences de température $T=T_{\mathrm{B}}-T_{\mathrm{A}}$ restent toujours suffisamment faibles ( $10^{-3}$ degrés) quel que soit $T<1000 \mathrm{~K}$, pour être négligées dans le rapport $k_{\lambda}$.

4.3 Egalité Des faisCEAUX ET SENSIBILITÉ DE L'APPAREIL. - Pour apprécier l'incertitude sur la comparaison des émissivités dues à l'appareil, nous avons monté un échantillon aux deux faces identiques. Les signaux $E_{\mathrm{A}}$ et $E_{\mathrm{B}}$ doivent être égaux quel que soit l'angle d'émission $\theta$ et quelle que soit la température étudiée. Les vérifications sur l'acier inoxydable montrent des différences de 3,5\% sur le rapport des facteurs d'émission $k_{\lambda}$. En faisant pivoter sur l'axe longitudinal l'échantillon de $180^{\circ}$, on vérifie que les faibles variations entre $E_{\mathrm{A}}$ et $E_{\mathrm{B}}$ sont bien liées à la mesure et non à des différences d'émissivité entre ces surfaces (la face $\mathrm{A}$ vue par le circuit $\mathrm{B}$ est comparée à l'analyse de la face A vue par le circuit A).

D'autre part, nous avons simulé de faibles variations d'émissivité en fonction de la longueur d'onde entre chacune des faces A et B en plaçant un filtre fin de polystyrène sur le trajet d'un des circuits. De cette manière pour un ruban d'acier ASI 304 à la température de $1000 \mathrm{~K}$ des variations de $k$ inférieures à $4 \%$ ont pu être enregistrées de façon reproductible entre 2 et $4 \mu \mathrm{m}$ et $\theta=0$, soit en supposant une émissivité moyenne de l'acier inoxydable lisse de 0,2 des variations $\Delta \varepsilon$ inférieures à 0,008 (Fig. 6).

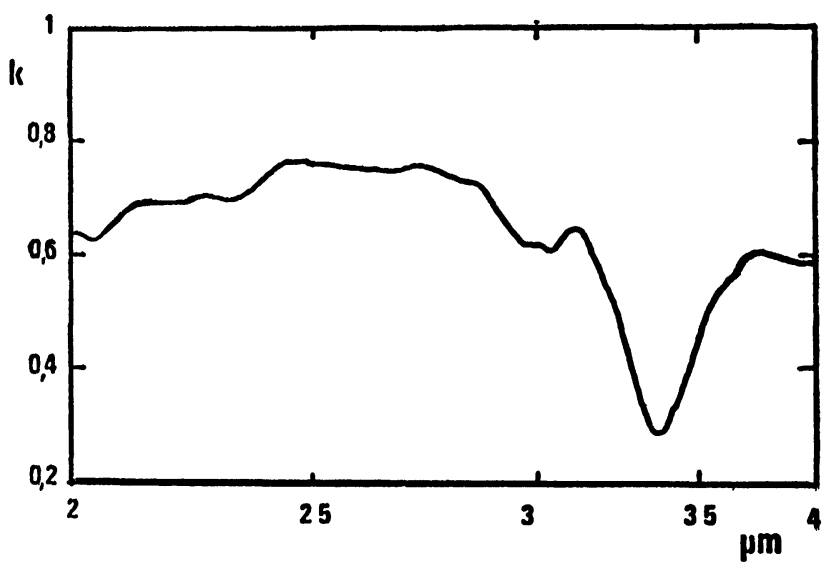

Fig. 6. - Variation de $k=E_{\mathrm{A}} / E_{\mathrm{B}}$ lorsqu'un filtre polystyrène est placé sur l'un des faisceaux.

[Variation of $k=E_{\mathrm{A}} / E_{\mathrm{B}}$, when a polystyrene is put on one of the beams.]

5. Conclusion - Pour satisfaire aux quatre conditions liées à l'analyse de faibles variations d'émissivité monochromatique directionnelle en fonction de paramètres caractérisant une surface, nos efforts ont surtout porté sur l'élaboration de la source [11] et de la méthode de traitement du signal. (Les techniques d'analyse du rayonnement, monochromateur, détecteur sont à l'heure actuelle suffisamment précises et leur influence dans l'incertitude sur $k$ peut être négligée.)

Le montage que nous avons décrit, ne réalise pas 
directement une mesure absolue des émissivités mais permet en comparant des énergies du même ordre de grandeur, d'observer de façon quasi différentielle de très faibles variations du pouvoir émissif, engendrées par la modification d'un paramètre unique (température, angle d'émission, épaisseur d'un dépôt, concentration d'un dopant, taille et forme d'une rugosité). L'égalité des températures entre la surface de référence (paramètre fixé), et la surface échantillon (paramètre modifié), l'égalité des angles d'émission, l'égalité des caractéristiques physicochimiques et du passé mécanique et thermique de leur substrat et parce que le temps entre les deux mesures face A, face B est faible
$(0,5 \mathrm{~s})$, la possibilité de négliger les modifications de la surface (dues par exemple aux variations de composition chimique locales, aux modifications cristallographiques ou à l'autodiffusion), font que les conditions expérimentales peuvent être considérées comme identiques lors de l'établissement de chaque rapport $\left(E_{\mathrm{A}} / E_{\mathrm{B}}\right)_{\lambda}$.

Ce montage expérimental de grande sensibilité reste néanmoins suffisamment pratique d'emploi (facilité du montage, démontage et positionnement des échantillons) pour permettre des études systématiques. Cet avantage sur les procédés expérimentaux existants est en grande partie due à l'originalité du support sources.

\section{Bibliographie}

[1] Touloukian, Y. S., DewitT, Thermophysical Properties of Matter (Plenum N-Y Washington) 1970, Vol. 7.

[2] Ody Sacadura, J. F., Int. J. Heat Mass Transfer 15 (1972) $1451 / 1465$.

Ody SaCadura, J. F., Rieutord, E., Communication présentée au VIe Congrès International sur le transfert de chaleur, Toronto, août 1978.

[3] Pigeat, Ph., Thèse Docteur-Ingénieur, Nancy (1980).

[4] Herve, P., Thèse Docteur ès Sciences, Paris-VI (1977).
[5] Devos, J.-C., Physica XX (1954) 690/714.

[6] Tennyson, S., J. Opt. Soc. Am. 67 (1977) 48-53.

[7] Habraken, L., Shungu, T. D., Leroy, U., Le Vide, les couches minces, $\mathrm{n}^{\circ} 199$ (1979) 385/429.

[8] Wieting et De Rosa, J. Opt. Soc. Am. 50 (1979) 1071/ 1078.

[9] Quinn, T. J., Brit J. Appl. Phys. 16 (1965) 873/980.

[10] Sievers, A. J., J. Opt. Soc. Am. 68 (1978) 1505/1516.

[11] Pigeat, Ph., Brevet d'invention no 80-02201.

[12] Nivort, M., publication interne au laboratoire. 